\title{
AUTOMATED IDENTIFICATION OF DIABETIC RETINOPATHY USING ALEXNET ARCHITECTURE
}

\author{
Hasirunnisa Shaik ${ }^{1}$, S. Deva Kumar ${ }^{2}$, M.Gargi ${ }^{3}$ and DS Bhupal Naik ${ }^{4}$ \\ ${ }^{1,3}$ Department of Computer Science \& Engineering, VLITS, A.P, India \\ ${ }^{2,4}$ Department of Computer Science \& Engineering, VFSTR, A.P, India \\ ${ }^{1}$ shaik.hasirunnisa77@gmail.com, ${ }^{2}$ deva.248@gmail.com, \\ ${ }^{3}$ gargilucky@gmail.com, ${ }^{4}$ dsbhupal@gmail.com
}

\begin{abstract}
Diabetic Retinopathy (DR) is one of the reasons for causing blindness globally. DR is a retinal disease that is found in diabetic patients. As performing retinal screening examinations is a time taken and unmet need on all diabetic patients, automated medical image analysis helps in identifying the disease severity. In this paper we study about the image classification using deep learning. The purpose of this study was to develop automated diagnostic technology for DR screening. In this paper we used AlexNet design with convolutional neural networks for DR classification. Here our algorithm processes all the fundus images and classified them as affected or not. A total of 800 images were taken from MESSIDOR dataset to train and test a model to separate non-affected from those which are affected. By using this algorithm our model get good results in Accuracy.
\end{abstract}

Keywords - AlexNet, Diabetic Retinopathy, Convolutional Neural Networks, Image Classification

\section{INTRODUCTION}

Diabetes is one of the wide spreading diseases around the globe and especially in India. There are many diseases related to Diabetes such as Diabetic Retinopathy (DR), nephropathy and neuropathy etc. The fluctuations in blood vessels of the eye origin results in irregularities. If these impediments are not focused within a period of time, this could tend to a huge number of incapacity to the patient which in turn causes expensive price and heavy work load for the consultants and the administration. Henceforth, it is essential to introduce an autonomous diagnostic technique to accelerate the job of the physician and minimize sickness of the patients. DR injures the blood vessels present in the retina. Frequently, it influences the eyes and could tend to exponential harm to the vision if not diagnosed appropriately.

On $14^{\text {th }}$ November, due to the occasion of world diabetes day, World Health Organization (WHO) stated that millions of people universally have diabetes. India has 31.7 million diabetic issues, and the quantity is predicted to rise to an overwhelming of 79.4 million by 2030. Pertaining to the investigation done, partial ophthalmologists that are present worldwide are positioned in six different countries such as China, USA, Russia, Japan, Brazil and India.

To analyze DR, a retinography is accomplished that comprises of seizing images interior to the eye (retina) through enlarging the pupil (mydriatic) or deprived of enlargement. Typically, ophthalmologists identify DR depending on features. Hemorrhages and MAs are the initially noticeable damages that specify a diabetic retinopathy and that are called as red lesions. Methodical screening through eye care

Received: July 16, 2019

Reviewed: October 10, 2019

Accepted: October 28, 2019 
experts is a cost operational health care exercise that could analyze the illness in the preliminary phase. So as to accommodate the screening and yearly evaluations obligates a huge amount of patients; an automatic screening device is a beneficial assistant to diabetes clinics. Presently, there are numerous approaches that could precisely diagnose definite DR associated injuries.

As the features which are mentioned above, to find out DR we need to develop an automated technique for the quick valuation of the retinal images.

\subsection{ORGANISATION OF THE PAPER}

A Brief introduction to Diabetic Retinopathy and cause of different diseases due to Diabetes are given along with the motivation for the proposed automated system. A detailed description of existing methodologies for the automatic detection of Diabetic Retinopathy is given in Section 2. The suggested detection of Diabetic Retinopathy using AlexNet architecture is briefly explained in section 3 . The experimental outcomes and its brief analysis is specified in section 4 followed by Conclusions and References given in Section 5 and Section 6.

\section{LITERATURE SURVEY}

Diabetes is one of the health problems that are found worldwide. Diabetes affects millions of people every year [1]. The disease that affects the fine vessels of blood present in retina is referred as Diabetic Retinopathy (DR) which is found in Diabetic patients. DR is one of the wide spreading disease by which blindness will occur if it's not recognized in its early stage. Only few DR patients are aware of their condition [2]. Although DR is a preventable disease [3].

DR is categorized into five stages according to its severity scale [16]. As if the severity of DR increases it may affect brain, heart and kidney. So it's necessary to know the severity of the disease [4]. DR prevention remains challenging for ophthalmologists because to find out the disease presence in patients directly is time taken and expensive [5]. DR early detection and severity diagnosis also remain subjective, as these were recorded in previous studies [6,7].

As direct examination is somewhat difficult Seoud et al. [19] developed a computeraided system for the fundus images classification. Image classification helps in training the computer with the data by differentiating the image into the prescribed category. Motivation by [20], here we study about image classification using deep learning. Here the image is processed in several steps such as pre-processing, segmentation and feature extraction [8]. The edge detection of an image is also done in image processing [9].

As deep learning is related to neural networks, A Neural Network is a network which functions same as a human brain. In this the neurons are the mathematical functions which takes the input, do some operations and gives the result [22].

Before knowing about Deep learning, there is a basic neural network called Artificial Neural Network (ANN). ANN's are also used in identifying the disease severity and to minimize the error function [18]. ANN's can map and capture all the features of an image [23] where as coming to Deep learning (DL), it is a subset of machine learning. DL is based on layered structure same as artificial neural network (ANN) with a little bit difference in the last layer. This makes deep learning different from other machine learning models [24,25]. DL is one of the most efficient model in machine learning and can solve the problems easily by extracting the features [17].

In deep learning, the neural network identifies the features of the image. Here we proposed a fully automated algorithm to identify DR from RGB images using Convolutional neural networks (CNN) which is a class of deep learning through the AlexNet architecture. Deep learning is used to characterize DR in the past [12-15]. The RGB images are processed based on their pixels of the image [21]. Here we developed an 
automated diagnostic system based on deep learning to classify the 800 DR images of MESSIDOR dataset [10]. These images are given as input and processed by our algorithm to predict a classification of 0 or 1 , where 0 is referred to no retinopathy and 1 referred to DR of any severity [11]. As our algorithm is automated in identifying the affected or non-affected images our solution gives better results in Accuracy.

\section{PROPOSED METHODOLOGY}

As DR is the leading cause of blindness, to overcome this we had introduced an automated DR detection using deep neural networks. In the first section [3.1] we had given a brief description about neural networks. Later in second section [3.2] we had described about the architecture on which we had processed our dataset.

\subsection{ARTIFICIAL NEURAL NETWORK}

Artificial Neural Networks (ANN) is based on Biological Neural Networks. In ANN each connected node is referred as a Neuron. Here the ANN works same as a biological brain, it receives the signal, process it and can signal a neuron connected to it. Here as stated above each neuron process one output which is send as input to the next neuron. A neuron can have multiple input and output connections. Each connection is assigned a weight. The neurons are typically organized into multiple layers, especially in deep learning.

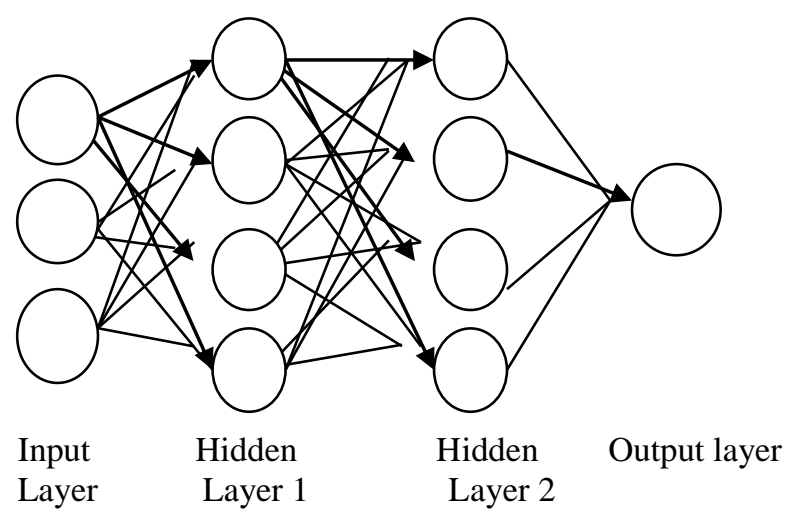

Fig. 1 Basic Deep Neural Network

As shown in the above Figure 1, if you send an image as input it will undergo many steps and then send an output. Between input and output layers there are some other layers which are referred as hidden layers. Among all these Convolutional Neural Network (CNN) is the best algorithm used for implementing deep learning techniques. $\mathrm{CNN}$ is also a kind of ANN. CNN is also known as ConvNet, consisting several layers named as convolutional layers, ReLu layer, pooling layer, and fully-connected layer.

\subsection{ALEXNET}

The Convnet is categorised into LeNet and AlexNet. Both these are pre-trained models. The LeNet is a Shallow Convolutional Neural network consists of convolutional layers, sub sampling layers, hidden layers and output layer. Whereas the AlexNet is a deep convolutional neural network consists of convolutional layers, sub sampling layers and fully connected layers. By using AlexNet we can train more than a million images from the database. 


\subsubsection{CONVOLUTION LAYER:}

A convolution layer is the first and basic step in CNN. In this layer we extract some features from the input image. These extracted features are termed as filter. This filter is scrolled all over the image and the dot product is stored in a feature map. Not only one filter is chosen similarly other filters are also taken from the input image and the same process is done to see the difference and the result.

\subsubsection{RELU LAYER:}

The Rectified Linear Unit is an activation function. In this function the negative value is replaced by 0 whereas the positive value is kept as same.

\subsubsection{POOLING LAYER:}

Pooling layer is also called as sub sampling layer. Pooling is of three types: Max. Pooling, Min. Pooling and Avg. Pooling. This pooling layer reduces the size of the image to perform Max. Pooling on the image firstly select one stride. Next scroll the stride over the filtered image and from that select one Max. Value and store it in the feature map. By performing this operation the dimensionality of the image reduces. Similarly to perform Min. or Avg. Pooling the same process is done.

\subsubsection{FULLY CONNECTED LAYER:}

This is the last layer in CNN. In this layer all the neurons have connected to the activations present in the previous layer. The neurons present in this layer capture elements of the input image and then predict it.
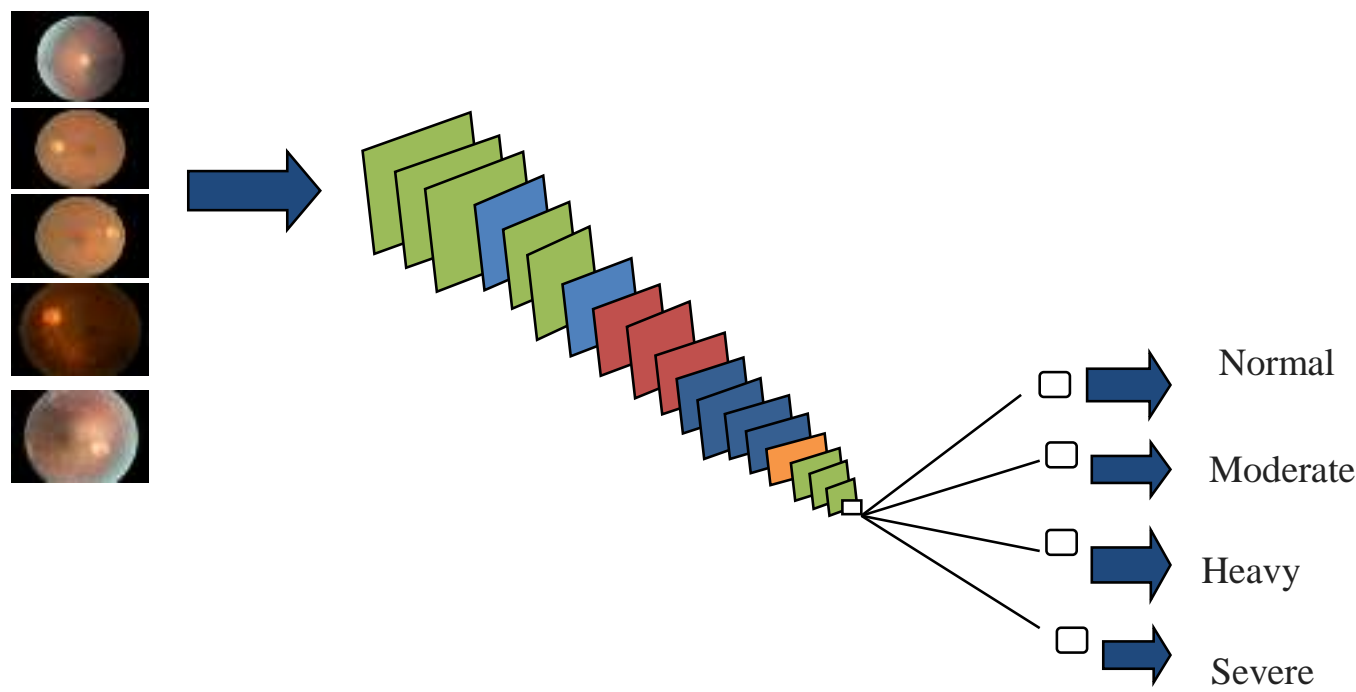

Fig. 2 Model training and evaluation

In the above Figure 2, as we send the images as input they had gone under several layers operations and then the final result is classified through the classification layer whether the image is affected or not and its stage severity is declared.

\section{EXPERIMENTAL RESULTS AND ITS ANALYSIS}

AlexNet architecture is one of the deep neural network with input size $227 \times 227$ was used to build the classification network. The network was pre-trained on the MESSIDOR dataset with 800 images for classification on the fundus images. This network was 
implemented by MATLAB R2018b framework. The inputs of the network are the fundus images and the output indicates the category of the input image. We processed our dataset on other pre-trained models like vgg1 and vgg19 for comparing the accuracy among them.

\subsection{PERFORMANCE EVALUATION}

$\mathrm{TP}=$ True Positive

FN $=$ False Negative

$\mathrm{TN}=$ True Negative

$\mathrm{FP}=$ False Positive

Accuracy test is used to differentiate the proportions of the affected cases and the unaffected cases in all cases.

$$
\text { Accuracy }=\frac{T P+T N}{T P+T N+F P+F N}
$$

Table 1. Accuracy of Pre-trained models

\begin{tabular}{|c|c|}
\hline Model & Accuracy \\
\hline VGG-19 & $90.16 \%$ \\
\hline VGG-16 & $92.35 \%$ \\
\hline AlexNet & $94.81 \%$ \\
\hline
\end{tabular}

\subsection{RESULTS \& DISCUSSIONS}

As we had tested our dataset on different pre-trained models, we get different accuracies. Let's have a brief description about those models.

When we processed our dataset on VGG-19, we got an accuracy of $90.16 \%$, this model consists of 19 layers and classify the images into categories. This model has an input size of $224 \times 224$. Here we had taken 800 images of MESSIDOR dataset, but the VGG-19 has an ability to be processed on millions of images. Here we had taken 20 epochs to classify the images and run the process on a single CPU system. Fig 3: Shows the accuracy of VGG-19.

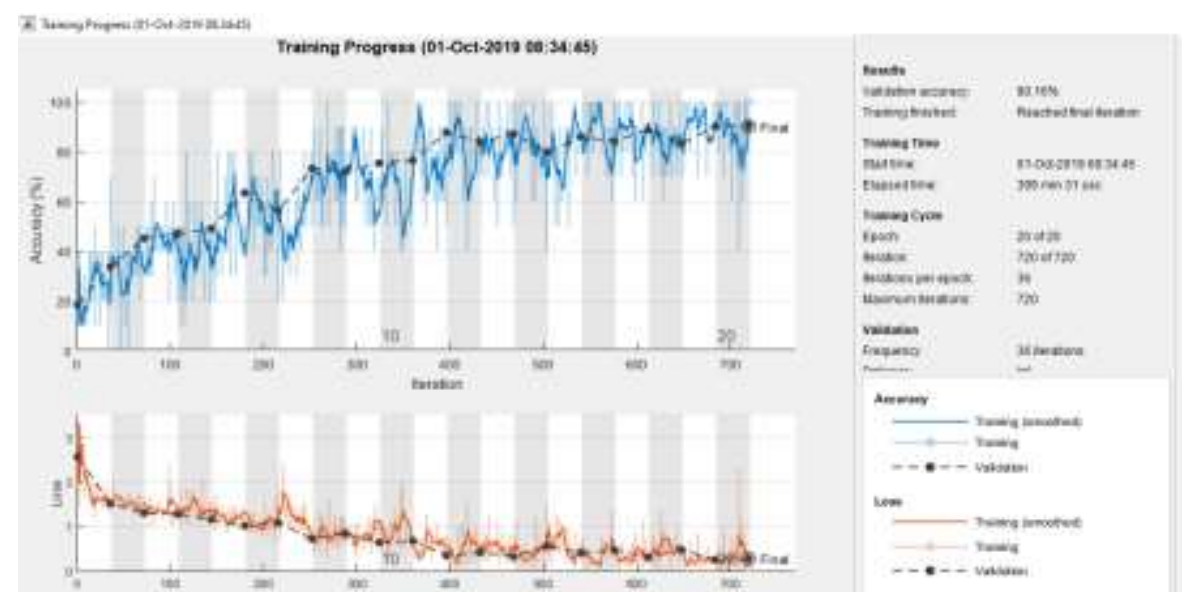

Fig. 3 VGG-19 
When we processed our dataset on VGG-16, we got an accuracy of $92.35 \%$, this model is also referred as OxfordNet and consists of 16 layers. This model has an input size of $224 \times 224$. Here we had taken 800 images of MESSIDOR dataset, but the VGG-16 has an ability to be processed on millions of images. Here we had taken 20 epochs to classify the images and run the process on a single CPU system. Fig 4: shows the accuracy of VGG16.

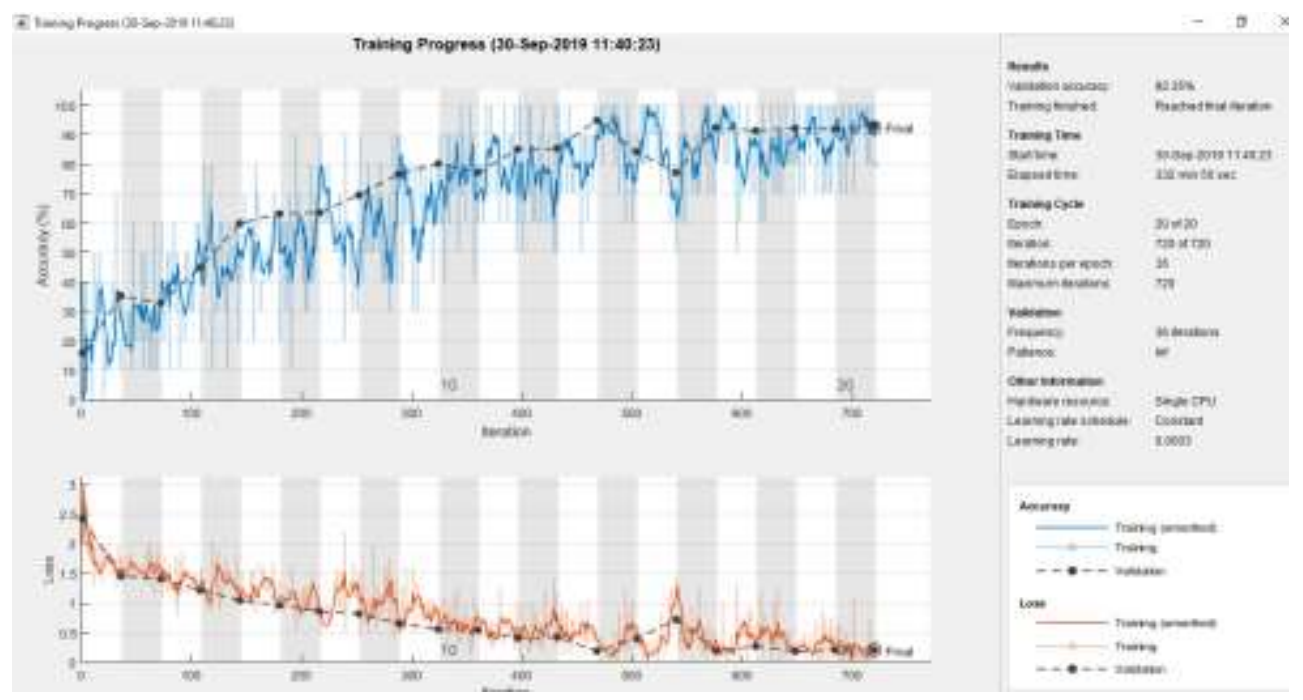

Fig. 4 VGG-16

When we processed our dataset on AlexNet, we got an accuracy of $94.81 \%$, this model has an input size of $227 \times 227$. Here we had taken 800 images of MESSIDOR dataset, but the AlexNet has an ability to be processed on millions of images. Here we had taken 20 epochs to classify the images and run the process on a single CPU system. Fig 5 and Fig 6 shows the accuracy and confusion matrix of AlexNet.

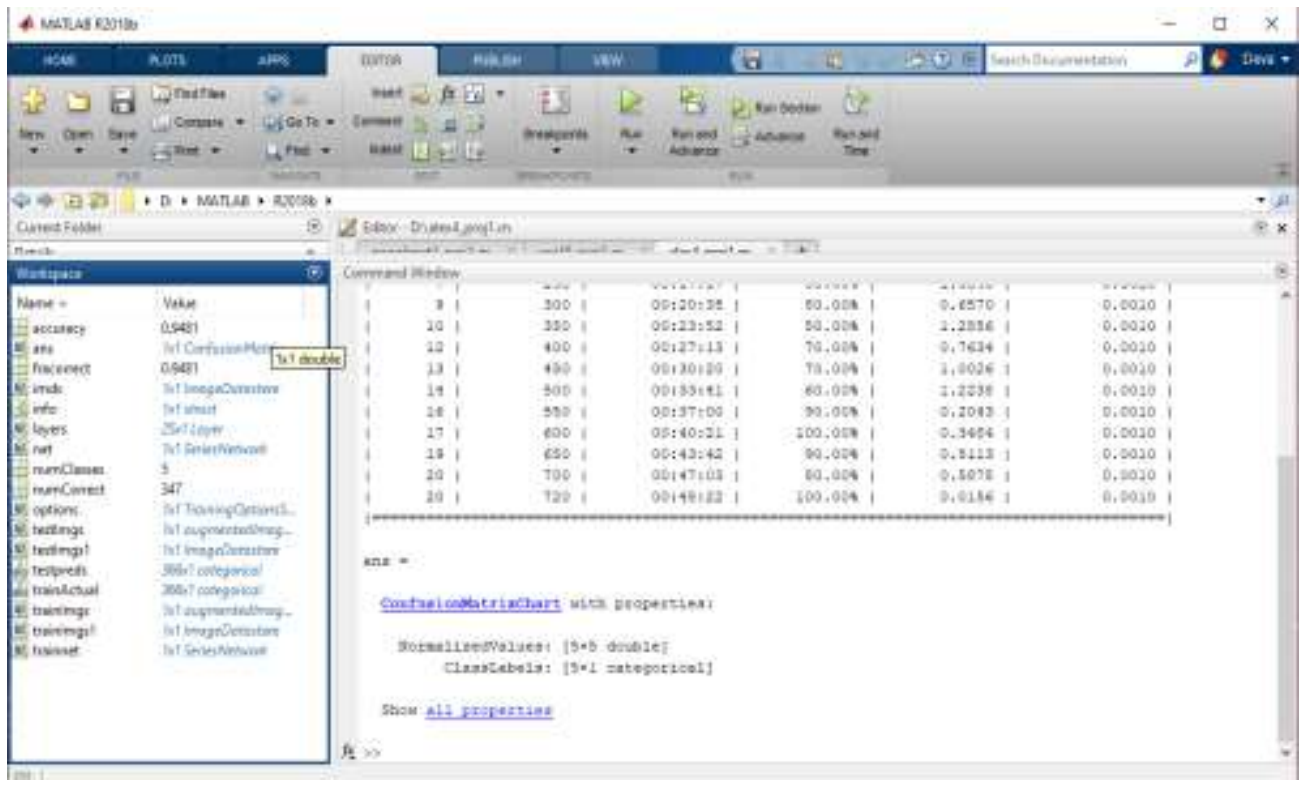

Fig 5 AlexNet 


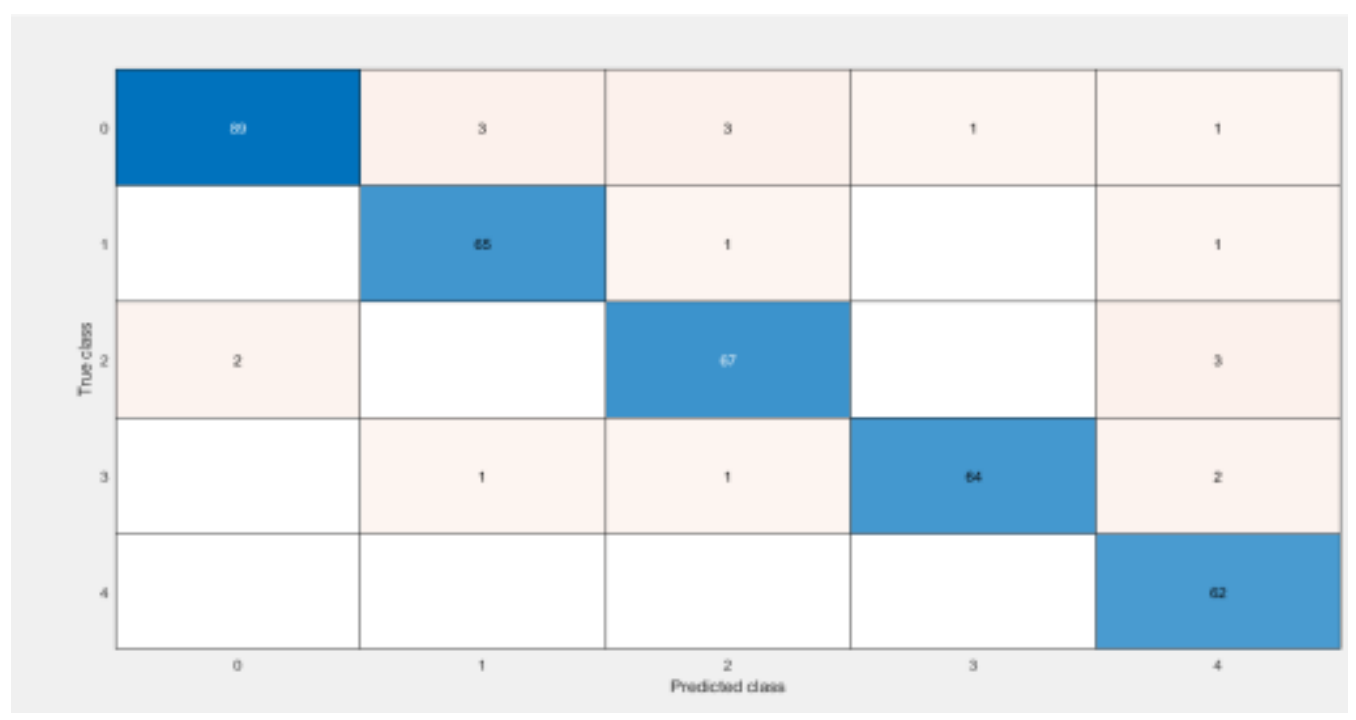

Fig 6 Confusion Matrix of AlexNet

Based on all the models performance AlexNet consumes a very less time compared to others, As we designed our model to help the Ophthalmologists in finding the disease severity, to reduce time, cost and to make patients to cure their disease in early stage so that they will have a bright vision.

\section{CONCLUSION}

As DR is eventually been found in maximum of Diabetic patients, its detection through manually is a time taken process. As the prevention of DR is a challenge for ophthalmologists, to overcome this drawback and to detect DR in a very less time we investigated an automated grading for DR using deep neural networks. Our pre-trained model gained accuracy of $94.81 \%$ and consumes very less time compared to other models. In future, we will run our model on Koggle dataset which contains thousands of images to improve the accuracy.

\section{REFERENCES}

[1] International Diabetes Federation (IDF). IDF Diabetes Atlas 7th edition; 2015. http://www.diabetesatlas.org/. Accessed, October 20, 2016.

[2] Beagley J, Guariguata L, Weil C, Motala AA. Global estimates of undiagnosed diabetes in adults. Diabetes Res Clin Pract.2015;103:150-160.

[3] Wilkinson CP, Ferris FL, Klein RE, et al. Proposed international clinical diabetic retinopathy and diabetic macular edema disease severity scales. Ophthalmology. 2003;110:1677-1682.

[4] J. Jayashree, J. Vijayashree and N. Ch. S. N. Iyengar, "Risk Level Prediction of Diabetic Retinopathy Using Adaptive Neural Fuzzy Inference System", International Journal of Advanced Science and Technology, SERSC Australia, ISSN: 2005-4238 (Print); 2207-6360 (Online), vol. 120, November (2018), (pp. 59-72), http://dx.doi.org/10.14257/ijast.2018.120.05.

[5] Ozieh MN, Bishu KG, Dismuke CE, Egede LE. Trends in health care expenditure in U.S. adults with diabetes:2002e2011. Diabetes Care. 2015;38:1844-1851.

[6] Sellahewa L, Simpson C, Maharajan P, et al. Grader agreement, and sensitivity and specificity of digital photography in a community optometry-based diabetic eye screening program. Clin Ophthalmol. 2014;8:1345-1349.

[7] Ruamviboonsuk P, Wongcumchang N, Surawongsin P, et al. Screening for diabetic retinopathy in rural area using single-field, digital fundus images. J Med Assoc Thail. 2005;88:176-180.

[8] Debnath Bhattacharyya and N. Thirupathi Rao, "Detection of Cyst in Human Brain Using Scan Images", International Journal of Advanced Science and Technology, NADIA, ISSN: 2005-4238 (Print); 2207-6360 (Online), vol. 124, March (2019), (pp. 111-120), http://dx.doi.org/10.33832/ijast.2019.124.10.

[9] Sanjay Yadav and A. K. Thripati, "Comparative Analysis of Canny Edge based Image Steganography with RSA Encryption", International Journal of Advanced Science and Technology, SERSC Australia, 
ISSN: 2005-4238 (Print); 2207-6360 (Online), vol. 104, July (2017), pp. 43-52, http://dx.doi.org/10.14257/ijast.2017.104.05.

[10] DongYue Wang and TaegKeun Whangbo, "Automatic Diagnostic System for Parkinson's Disease Based on Deep Learning using Midbrain Magnetic Resonance Images", International Journal of Advanced Science and Technology, NADIA, ISSN: 2005-4238 (Print); 2207-6360 (Online), vol. 124, March (2019), (pp. 1-20), http://dx.doi.org/10.33832/ijast.2019.124.01.

[11] Mohammad Shah Alamgir and Sumit Dev, "Design and Implementation of an Automatic Voltage Regulator with a Great Precision and Proper Hysteresis", International Journal of Advanced Science and Technology, SERSC Australia, ISSN: 2005-4238 (Print); 2207-6360 (Online), vol. 75, February (2015), pp. 21-32, http://dx.doi.org/10.14257/ijast.2015.75.03.

[12] LeCun Y, Kavukcuoglu K, Farabet C. Convolutional networks and applications in vision. ISCAS 2010d2010 IEEE International Symposium on Circuits and Systems. Nano-Bio Circuit Fabrics and Systems; 2010:253-256.

[13] Lecun Y, Bengio Y, Hinton G. Deep learning. Nature. 2015;521:436-444.

[14] Kaggle, Inc. Diabetic retinopathy detection; 2015. https://www.kaggle.com/c/diabetic-retinopathydetection.Accessed 1.12.2016.

[15] Cuadros J, Bresnick G, Affiliations A. EyePACS: an adaptable telemedicine system for diabetic retinopathy screening. J Diabetes Sci Technol. 2009;3:509-516.

[16] Gulshan, V.; Peng, L.; Coram, M.; Stumpe, M.C.;Wu, D.; Narayanaswamy, A.; Venugopalan, S.;Widner, K.; Madams, T.; Cuadros, J. Development and validation of a deep learning algorithm for detection of diabetic retinopathy in retinal fundus photographs. JAMA 2016, 316, 2402-2410. [CrossRef] [PubMed]

[17] Raju Anitha, S. Jyothi, Venkata Naresh Mandhala, Debnath Bhattacharyya and Tai-hoon Kim, "Deep Learning Image Processing Technique for Early Detection of Alzheimer's Disease", International Journal of Advanced Science and Technology, SERSC Australia, ISSN: 2005-4238 (Print); 2207-6360 (Online), vol. 107, October (2017), pp. 85-104, http://dx.doi.org/10.14257/ijast.2017.107.07.

[18] Nesreen Samer El-Jerjawi and Samy S. Abu-Naser, "Diabetes Prediction Using Artificial Neural Network", International Journal of Advanced Science and Technology, SERSC Australia, ISSN: 20054238 (Print); 2207-6360 (Online), vol. 121, December (2018), (pp. 55-64), http://dx.doi.org/10.14257/ijast.2018.121.05.

[19] Seoud, L.; Chelbi, J.; Cheriet, F. Automatic grading of diabetic retinopathy on a public database. In Proceedings of the Ophthalmic Medical Image Analysis Second International Workshop, Munich, Germany, 9 October 2015.

[20] https://in.mathworks.com/matlabcentral/fileexchange/59133-neural-network-toolbox-tm--model-foralexnet-network.

[21] Suolan Liu, Chen Yue and Hongyuan Wang, "An Improved Hybrid Encryption Algorithm for RGB Images", International Journal of Advanced Science and Technology, SERSC Australia, ISSN: 20054238 (Print); 2207-6360 (Online), vol. 95, October (2016), pp. 37-44, http://dx.doi.org/10.14257/ijast.2016.95.04.

[22] Ankita Wadhawan and Avani Bhatia, "Neural Network Based Intelligent Retrieval System for Verifying Dynamic Signatures", International Journal of Advanced Science and Technology, SERSC Australia, ISSN: 2005-4238 (Print); 2207-6360 (Online), vol. 83, October (2015), pp. 27-40, http://dx.doi.org/10.14257/ijast.2015.83.03.

[23] Lyamine Briki and Kamel Djeghaba, "A New Plasticity Model for Concrete in Compression Based on Artificial Neural Networks", International Journal of Advanced Science and Technology, SERSC Australia, ISSN: 2005-4238 (Print); 2207-6360 (Online), vol. 75, February (2015), pp. 43-50, http://dx.doi.org/10.14257/ijast.2015.75.05.

[24] Deep Learning with MATLAB - matlab expo2018.

[25] Introducing Deep Learning with the MATLAB - Deep Learning E-Book provided by the mathworks. 